\title{
Investigations of Peripheral Propulsive Deceleration Jets on a Mars Science Laboratory Aeroshell
}

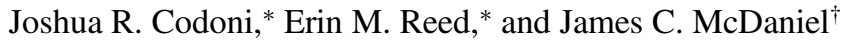 \\ University of Virginia, Charlottesville, Virginia 22903 \\ and \\ Hicham Alkandry \\ University of Michigan, Ann Arbor, Michigan 48109
}

\begin{abstract}
DOI: $\underline{10.2514 / 1 . A 32456}$
The objective to send more massive landed missions to the surface of Mars necessitates further research and development for ways to adequately decelerate the lander, such as propulsive deceleration. Experimental measurements using planar laser-induced iodine fluorescence provide qualitative visualizations and quantitative propulsive decelerator jet mole fraction measurements over a $0.22 \%$ scaled Mars Science Laboratory aeroshell. Peripheral (off-centerline) sonic and supersonic propulsive decelerator jet models, with jet exit velocities of Mach 1.0 and 2.66, were studied in Mach 12 flow and compared with numerical results obtained using computational fluid dynamics. Experimental visualizations were obtained for various thrust coefficients ranging from 0.5 to 3.0 in increments of 0.5. Experimental results indicate that, for both sonic and supersonic jets, the bow shock is preserved between the peripheral jets and, as thrust coefficient increases, the bow shock is pushed farther from the aeroshell forebody, with the supersonic jets having a greater shock standoff distance than sonic models at thrust coefficient greater than 2.0. Comparisons of the experimental visualizations to computed streamlines and shock locations show good agreement. Computed propulsive decelerator jet mole fractions for both sonic and supersonic thrust coefficient of 1.5 closely match experimental results through the jet cores and in cross-sectional cuts.
\end{abstract}

\section{Nomenclature}

$A=$ empirically determined constant

$A_{e}=$ exit area of propulsive deceleration jet, $\mathrm{m}^{2}$

$C_{T}=$ coefficient of thrust

$D=$ orifice diameter, $\mathrm{mm}$

$M=$ Mach number

$x=$ distance from orifice, $\mathrm{mm}$

$x_{0}=$ empirically determined constant, $\mathrm{mm}$

$\gamma=$ specific heat ratio

$T=$ temperature, $\mathrm{K}$

$\mathrm{Th}=$ jet thrust, $\mathrm{N}$

$q=$ dynamic pressure, $\mathrm{Pa}$

$S=$ surface area of aeroshell, $\mathrm{m}^{s}$

$\dot{m}=$ mass flow rate, $\mathrm{kg} / \mathrm{s}$

$V=$ velocity, $\mathrm{m} / \mathrm{s}$

$p=$ pressure, $\mathrm{Pa}$

Subscripts

$e \quad=$ jet exit condition

$0=$ total condition

$\infty=$ freestream conditions

Presented as Paper 2011-1036 at the 49th AIAA Aerospace Sciences Meeting, Orlando, FL, 4-7 January 2011; received 11 July 2012; revision received 10 April 2013; accepted for publication 14 April 2013; published online 14 February 2014. Copyright $@ 2013$ by Joshua R. Codoni. Published by the American Institute of Aeronautics and Astronautics, Inc., with permission. Copies of this paper may be made for personal or internal use, on condition that the copier pay the $\$ 10.00$ per-copy fee to the Copyright Clearance Center, Inc., 222 Rosewood Drive, Danvers, MA 01923; include the code 1533-6794/14 and \$10.00 in correspondence with the CCC.

*Graduate Student, Department of Mechanical and Aerospace Engineering. Student Member AIAA.

${ }^{\dagger}$ Professor, Department of Mechanical and Aerospace Engineering. Associate Fellow AIAA.

${ }^{\ddagger}$ Graduate Student, Department of Aerospace Engineering. Student Member AIAA.

$\S$ James E. Knott Professor of Engineering, Department of Aerospace Engineering. Fellow AIAA.

\section{Introduction}

$\mathbf{E}$ NTRY, descent, and landing is one of many challenging aspects of Mars missions. A thin atmospheric density, roughly $1 \%$ of Earth's, causes significant heating, but insufficient deceleration for High Mass Mars Entry Systems (HMMES). Because of the thin atmosphere, as payload mass increases, it becomes a greater challenge to adequately slow the landing vehicle quickly enough to enter a landing configuration [1]. Therefore, as human-scale missions are planned on the scale of orders of magnitude larger than landers to date, and too large for traditional parachutes, it becomes necessary to explore new methods for decelerating landing vehicles.

Retropropulsion, or propulsive deceleration (PD), has recently received renewed interest as an enabling technology for adequately decelerating HMMES at supersonic and hypersonic Mach numbers [2]. Single or multiple PD jets are fired from the aeroshell against the freestream velocity, using the jet thrust to achieve the deceleration. To date, there is a dearth of experimental and computational data on multiple PD jets located around the periphery of the aeroshell [3]. With the exception of recent data [4] for peripheral three-nozzle configurations, the only available data for other peripheral configurations were collected in the late 1960s and early 1970s for freestream Mach numbers up to 6.0 using schlieren/shadowgraphs for visualizations and pressure taps/strain-gages for drag characteristics [5-7]. These previous experiments indicate that aerodynamic drag can be preserved to some extent while thrust force is increased for peripheral multiple jet configurations, unlike single-jet configurations where the drag asymptotically decreases with increasing jet thrust. The degree of drag preservation is greater than with a single PD jet on the aeroshell centerline. Preservation of aerodynamic drag with increasing thrust using peripheral PD jets shows promise for this technology as better enabling HMMES to decelerate. One recent study [8] finds that initiating PD jets at about Mach 5 can reduce propellant mass fraction by roughly $2.3 \%$, or $1200 \mathrm{~kg}$, for a ballistic coefficient (ratio of vehicle mass to drag, giving the ability of the vehicle to decelerate) of $400 \mathrm{~kg} / \mathrm{m}^{2}$, if partial drag is preserved by using a low coefficient of thrust $C_{T}$. However, there is still much to learn about the flow properties of the highly complex interaction of a supersonic/hypersonic freestream with sonic/supersonic peripheral PD jets. It is necessary to further 


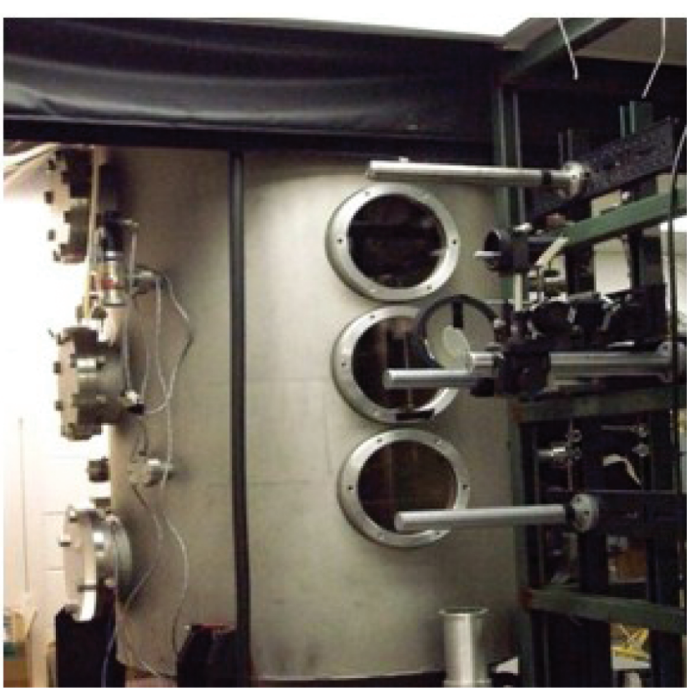

a) Vacuum chamber

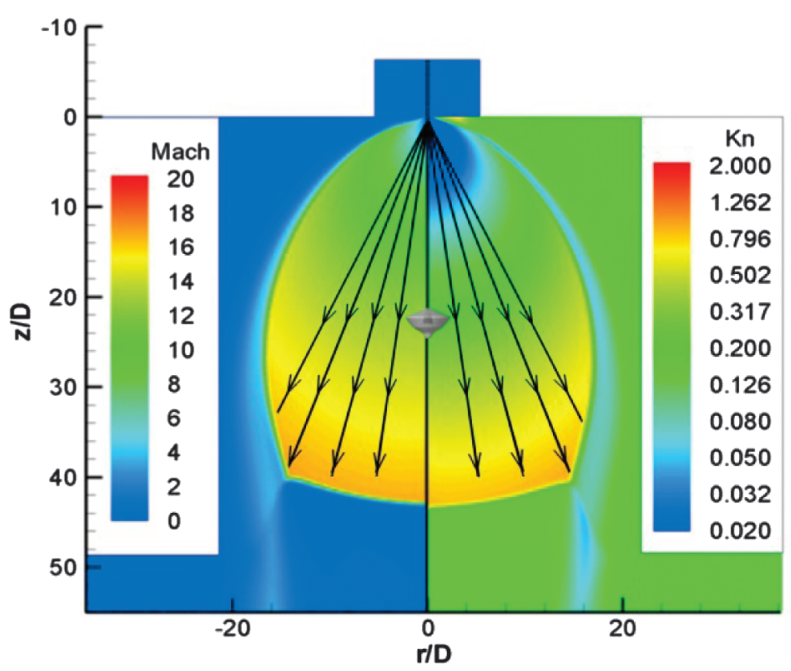

b) Hypersonic test section [9]

Fig. 1 Vacuum chamber and calculation of Mach and Knudsen numbers in hypersonic test section.

investigate this interaction to better optimize possible weight savings as well as optimal initiation conditions.

This paper presents current experimental work employing a technique known as planar laser-induced iodine fluorescence (PLIIF) to obtain visualization images and PD jet mole fraction measurements for sonic and supersonic peripheral four-jet PD models opposing a near-continuum freestream at Mach 12. Experimental visualizations and PD jet mole fractions will be compared with computational fluid dynamic (CFD) numerical results, and will provide a valuable set of data and calculations, providing insight on the highly complex flowfield of a retrorocket interacting with a hypersonic freestream flow.

\section{Experimental Technique}

A. Facilities

PD jet experiments are conducted using a continuous flow hypersonic wind tunnel. The wind tunnel uses a continuously evacuated vacuum chamber, shown in Fig. 1a, to provide the low back pressures necessary to produce the hypersonic test section flow. Low chamber back pressures are achieved using three vacuum pumps: a MicroVac pump, booster pump, and a high pressure pump, maintaining pressures on the order of 300 mtorr even when the main flow is introduced. The vacuum chamber has three portholes, which provide optical access for the collimated laser sheet necessary for PLIIF. A fourth porthole, not visible in the image, perpendicular to the laser sheet entry, provides optical access for a charge-coupled device (CCD) camera.

The test section of the hypersonic wind tunnel is an underexpanded jet. Nitrogen gas, typically seeded with approximately one part per million iodine at $1.8 \mathrm{~atm}$ and $297 \mathrm{~K}$ exhausts through a $2 \mathrm{~mm}$ sonic orifice into the continuously evacuated chamber. As shown in Fig. 1b, the jet expands from a point source and produces a barrel shock, terminating in a Mach disk approximately $8 \mathrm{~cm}$ downstream of the orifice. The isentropic core of the jet expansion provides a test section capable of Mach numbers from 1 to 16, and Knudsen numbers (ratio of mean free path to jet exit orifice diameter) from 0.02 to 1 . The Mach number versus distance from the sonic orifice is calculated using the Ashkenas and Sherman relationship, as shown in Eq. (1),

$$
M=A\left(\frac{x-x_{0}}{D}\right)^{\gamma-1}-\frac{1}{2}\left(\frac{\gamma+1}{\gamma-1}\right)\left[A\left(\frac{x-x_{0}}{D}\right)^{\gamma-1}\right]^{-1}
$$

where $x_{0}$ and $A$ are constants empirically determined by Ashkenas and Sherman for the specific heat ratio corresponding to $N_{2}$, the test section gas [10]. Mars Science Laboratory (MSL) models are placed along the centerline of the underexpanded jet at positions corresponding to the desired Mach number calculated with the Ashkenas and Sherman relationship. For the following work, testing is conducted at Mach 12 conditions, and the forebody of the model is placed $4.2 \mathrm{~cm}$ downstream of the $2 \mathrm{~mm}$ orifice. Using isentropic relations, the freestream conditions at Mach 12 are listed in Table 1 .

\section{B. Planar Laser-Induced Iodine Fluorescence Experimental Method}

PLIIF is an optical nonintrusive, time-averaged measurement technique that has been extensively developed and used for nearly 30 years $[9,11-14]$. PLIIF uses $I_{2}$ as the fluorescing species and is capable of producing planar measurements for flow visualization and quantitative measurement of mole fraction, velocity, pressure, density, and temperature. Quantitative velocity, pressure, density, and temperature measurements are obtained by operating the laser in narrowband mode, where the laser linewidth is much less than the iodine absorption linewidth, and by scanning over the iodine absorption transitions. Qualitative visualizations and quantitative mole fraction measurements can be obtained by operating the laser in broadband mode, where the laser linewidth is much greater than the iodine absorption linewidth. PLIIF is advantageous over other methods, such as schlieren and shadowgraph, due to its ability to provide sufficient signal for flowfield imaging even in rarefied regimes. Another benefit of PLIIF is the ability to produce accurate measurements across shocks, unlike other methods such as particle image velocimetry [15].

In this study, PLIIF is used to obtain qualitative visualization and quantitative mole fraction results. The experiment test section setup for the PLIIF method is pictured in Fig. 2. A laser beam from a Spectra-Physics Beamlok 2080A argon-ion laser operating at $514.5 \mathrm{~nm}$ is collimated into a thin laser sheet using a series of optics. The laser sheet propagates through the bottom porthole of the vacuum chamber and is incident on the top of the model after reflecting from two mirrors placed inside the chamber. Laser radiation incident on an iodine molecule will excite it to an upper energy state. The excited molecule can then return to the ground state via multiple processes, one of which is by emitting a photon, called fluorescence. The resulting iodine fluorescence is captured at $90 \mathrm{deg}$ to the laser sheet

Table 1 Freestream conditions

\begin{tabular}{lr}
\hline \hline Parameter & Value \\
\hline$M_{\infty}$ & 12 \\
$T_{\infty}, \mathrm{K}$ & 10 \\
$q_{\infty}, \mathrm{Pa}$ & 127 \\
$\operatorname{Re}_{\infty}$ & 1200 \\
\hline \hline
\end{tabular}


by a CCD camera for exposure times from 10 to $45 \mathrm{~s}$. Scattered laser light is blocked with a glass orange Heliopan \#22 filter.

\section{Model Design}

Visualization results for two models will be shown and discussed: a sonic peripheral four-jet model and a supersonic peripheral four-jet model, both with jets placed midway between the stagnation point and the model shoulder. The models are $0.22 \%$ scale of the MSL frontal aeroshell and constructed of aluminum. The jets for the sonic and supersonic models are oriented normal to the direction of freestream flow, which causes the jet exit orifice to be slightly elliptical. The sonic jet model has an equivalent jet exit diameter of $0.5 \mathrm{~mm}$ whereas the supersonic jet model has an equivalent jet exit diameter of $0.9 \mathrm{~mm}$ with a throat diameter of $0.5 \mathrm{~mm}$, corresponding to a jet exit Mach number of 2.66. A detailed sketch of each model geometry can be seen in [16]. Figure $\underline{3}$ shows the internals of the wind

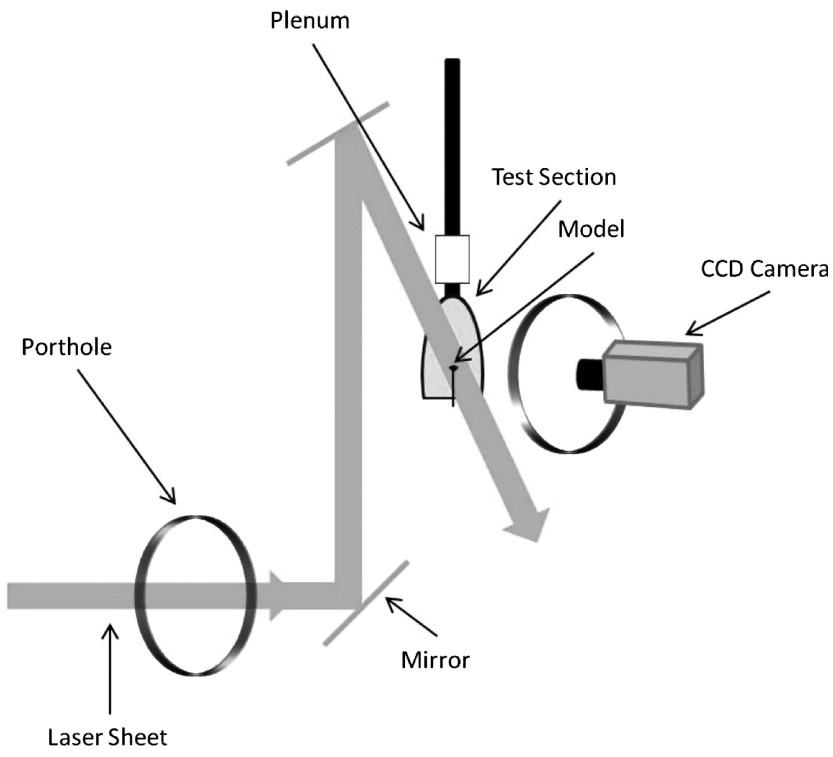

Fig. 2 PLIIF setup.

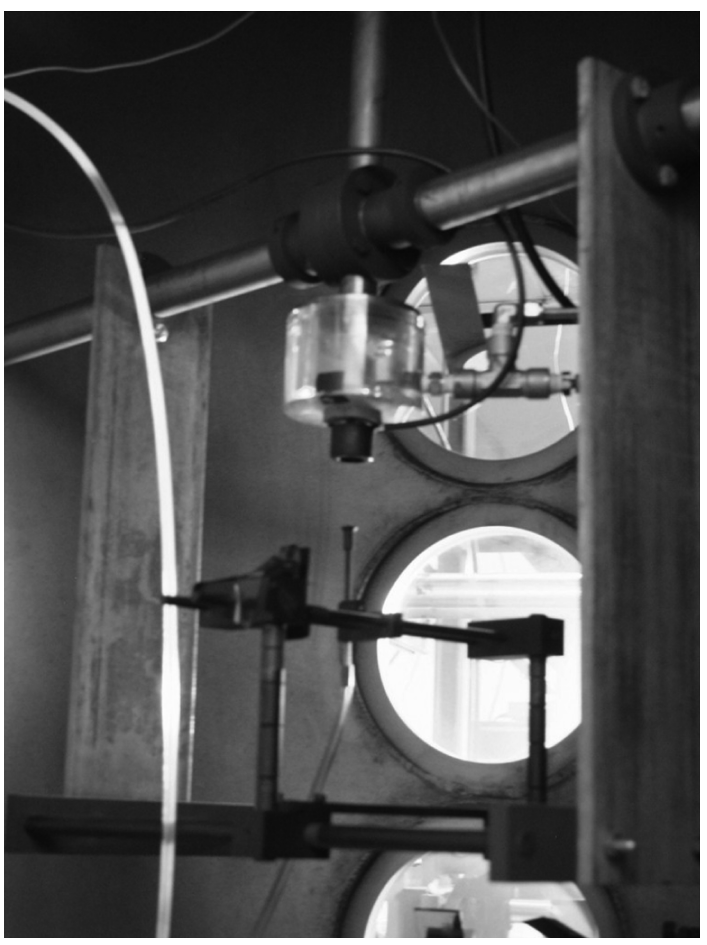

Fig. 3 Model mounted in vacuum chamber.
Table 2 Freestream conditions

\begin{tabular}{lcccccc}
\hline \hline & \multicolumn{3}{c}{ Sonic } & \multicolumn{3}{c}{ Supersonic } \\
\cline { 2 - 7 } $\begin{array}{l}\text { Thrust } \\
\text { coefficient }\end{array}$ & $T_{0}$ & $\begin{array}{c}p_{0}, \\
\mathrm{kPa}\end{array}$ & $\begin{array}{c}\dot{m}_{\text {ideal }}, \\
\mathrm{mg} / \mathrm{s}\end{array}$ & $T_{0}$ & $\begin{array}{c}p_{0}, \\
\mathrm{kPa}\end{array}$ & $\begin{array}{c}\dot{m}_{\text {ideal }}, \\
\mathrm{mg} / \mathrm{s}\end{array}$ \\
\hline 0.5 & 297 & 5.03 & 2.4 & 297 & 4.47 & 2.1 \\
1.0 & 297 & 10.05 & 4.6 & 297 & 8.51 & 4.0 \\
1.5 & 297 & 15.08 & 6.9 & 297 & 12.56 & 5.9 \\
2.0 & 297 & 20.11 & 9.2 & 297 & 16.60 & 7.8 \\
2.5 & 297 & 25.14 & 11.4 & 297 & 20.65 & 9.7 \\
3.0 & 297 & 30.16 & 13.7 & 297 & 24.69 & 11.6 \\
\hline \hline
\end{tabular}

tunnel, the freestream plenum is the clear acrylic cylinder in the top center of the image, and the model is directly below, supported via the sting by the traversing mounting system shown. The models, as well as most hardware subject to laser radiation, are painted matte black to minimize scattered light reflections from inside the chamber. Nitrogen seeded with iodine is supplied to the PD jets via a sting mounted to the aftbody of the model.

\section{Thrust Calculations}

To compare experimental data from other facilities and CFD results, a nondimensional $C_{T}$ is used. $C_{T}$, defined by McGhee as the ratio of jet thrust to the freestream dynamic pressure times the frontal area of the model, is as follows [17]:

$$
C_{T}=\frac{\mathrm{Th}}{q_{\infty} S}=\frac{\dot{m} V_{e}+\left(p_{e}-p_{\infty}\right) A_{e}}{q_{\infty} S}
$$

The thrust coefficient in Eq. (2) was calculated using isentropic relations and the Ashkenas and Sherman [Eq. (1)] relationship for the freestream conditions listed in Table 1 . The $\overline{P D}$ jet conditions for a single nozzle are listed in Table 2 , assuming inviscid flow for the calculation of mass flow rate.

\section{Results}

\section{A. Experimental Visualizations}

Figures 4 and 5 are experimental PLIIF visualizations of the sonic and supersonic peripheral four-jet PD models, respectively, at $C_{T}$ from 0.5 to 3.0 in increments of 0.5 . The following visualization images were taken with iodine seeded in the freestream and PD jet. Freestream flow is from the top of the images to the bottom. The forebody of the model MSL aeroshell is placed at the Mach 12 location in the hypersonic underexpanded jet test flowfield. The model is superimposed in the PLIIF images to indicate the geometry of the image. In these images, only two of the four jets are visible because the laser sheet passes through the center of two jets only. Furthermore, the flowfields are symmetric, and so the images are mirrored about the model centerline to remove the Doppler shift effect, which is otherwise observed in the fluorescence images.

The sonic PD jets (Fig. 4) are underexpanded jets much like the hypersonic test flowfield. The PD jets exit the orifice at Mach 1 and freely expand until they terminate in the jet shock. The bow shock is preserved above the stagnation region of the model forebody, but the PD jets cause the shock to be pushed away from the model forebody. Figure $\underline{5}$ is the supersonic peripheral four-jet PD model and has a jet exit Mach number of 2.66. Much like the sonic case, the bow shock is preserved above the stagnation region of the model but is pushed farther upstream than the sonic case due to greater penetration by the supersonic jets. Fluorescence below the PD jets and around the shoulder of the model are observed for both the sonic and supersonic cases up to $C_{T}=1.5$. The sonic PD jets have a greater jet turning angle than the supersonic jets.

Table 3 is a comparison of the shock standoff distance (SSD) and maximum upstream PD jet penetration distance for the sonic and supersonic test cases. In regions with Mach number greater than or equal to 6 , the fluorescence signal is directly proportional to the number density [18]. Thus, there is a gradient in fluorescence signal 

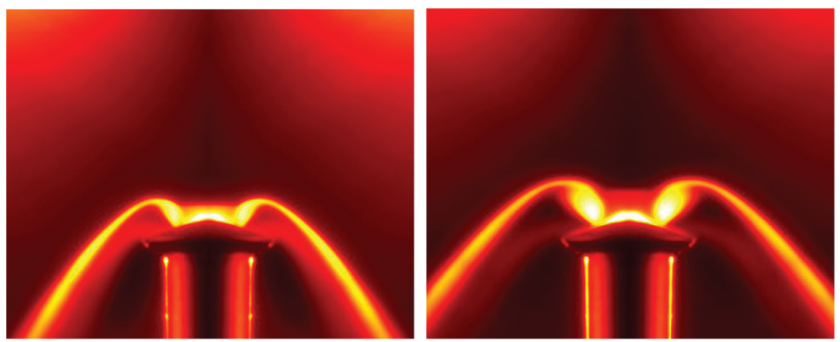

a) $\mathrm{C}_{\mathrm{T}}=0.5$

b) $\mathrm{C}_{\mathrm{T}}=\mathbf{1 . 0}$
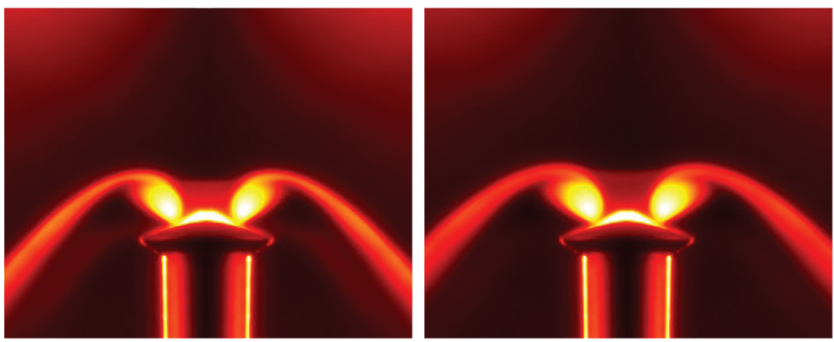

c) $C_{T}=1.5$

d) $\mathrm{C}_{\mathrm{T}}=\mathbf{2 . 0}$
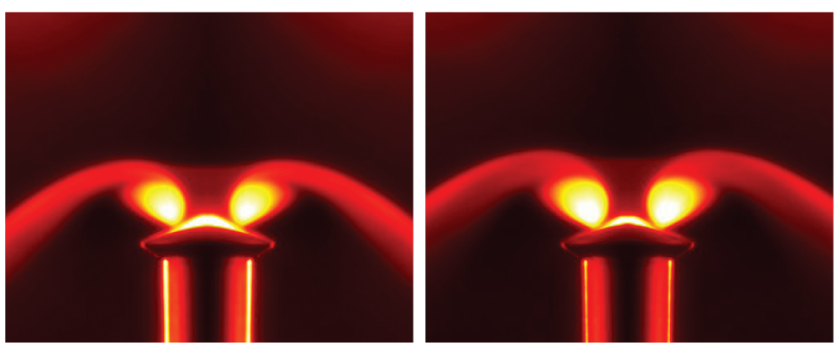

e) $C_{T}=2.5$

f) $\mathrm{C}_{\mathrm{T}}=\mathbf{3 . 0}$

Fig. 4 Sonic peripheral four-jet PD model, $M_{\text {jet }}=1.0$, for range of $C_{T}$ from 0.5 (a) to 3.0 (f).

as the flow begins to pass through a shock. The SSD is taken as the point where the fluorescence signal has increased by $10 \%$ above the background level. The SSD is normalized to the model frontal diameter for the sonic and supersonic cases. The uncertainty is measured as \pm 3 CCD camera pixels in the region between the shock and model surface locations, and \pm 3 in the model diameter. Combining these uncertainties for the ratio of SSD to model diameter results in an overall uncertainty of 0.03 for all cases and is indicated as a percentage uncertainty in the table. The standoff distance for the supersonic case is approximately the same as the sonic case until a $C_{T}$ of 1.5. For $C_{T}$ of 3.0, the shock standoff distance is about $18 \%$ greater for the supersonic case.

The same method is used to obtain the maximum PD jet penetration, which is the point farthest upstream where the shock begins to form directly upstream of the PD jet. The maximum PD jet penetration distance, normalized to the model aeroshell diameter, is shown in Table 3. The supersonic jets extend farther into the freestream than the sonic jets for all $C_{T}$ tested, even for small $C_{T}$ where the shock standoff is roughly the same. The penetration distance for the supersonic case is approximately $50 \%$ greater than the sonic case at $C_{T}=3.0$.

Quantitative mole fraction images were also obtained using PLIIF. By taking the ratio of a jet-only seeded image to a full-flow seeded image (Fig. 6a divided by Fig. 6b) and normalizing the ratio by the value in the $\overline{\mathrm{PD}}$ core where the jet mole fraction is unity, a jet mole fraction image results [18]. The fluorescence above the center of the model between the PD jets is only visible in the full-flow seeded case (Fig. 6b), which indicates the fluid in this region of the flowfield is primarily from compressed fluid behind the bow shock from the freestream.

The resulting mole fraction images are shown in Fig. 7 for the sonic and supersonic PD jet models for a $C_{T}$ of 1.5. The color contours give spatially resolved quantitative values of the local jet mole fraction,
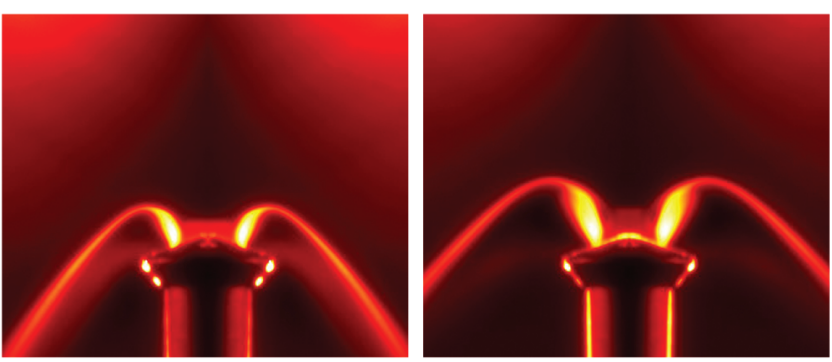

a) $\mathrm{C}_{\mathrm{T}}=0.5$

b) $\mathbf{C}_{\mathbf{T}}=\mathbf{1 . 0}$
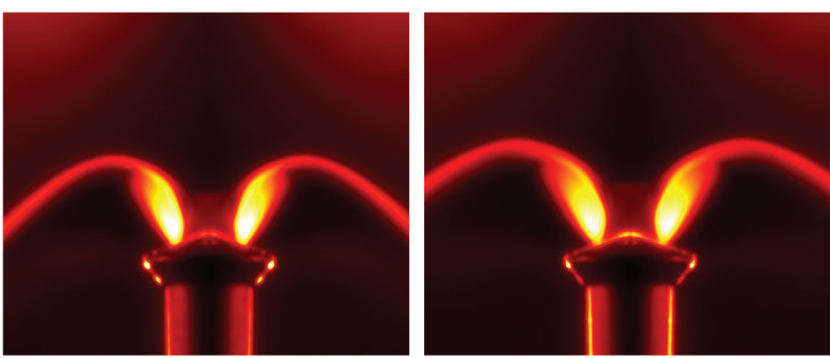

c) $\mathrm{C}_{\mathrm{T}}=1.5$

d) $C_{T}=2.0$
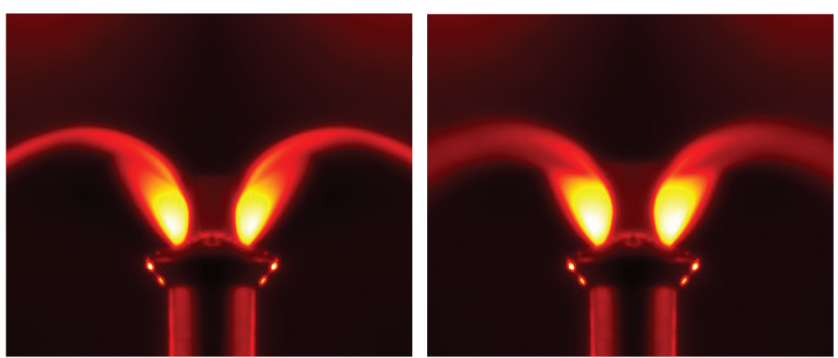

e) $\mathrm{C}_{\mathrm{T}}=2.5$

f) $\mathrm{C}_{\mathrm{T}}=\mathbf{3 . 0}$

Fig. 5 Supersonic periphery four-jet PD model, $M_{\text {jet }}=2.66$, for $C_{T}$ from 0.5 (a) to 3.0 (f).

which are due to the PD jet mixing with the Mach 12 freestream. These quantitative images provide the opportunity to validate CFD results, as will be shown in the next section of this paper.

\section{B. Numerical Simulation Comparisons}

Results from the experimental and CFD LeMANS calculations will be compared to assess the physical accuracy of the computations for the sonic and supersonic test cases discussed in [16]. Figures 8 and $\underline{9}$ are experimental images of the sonic and supersonic cases, respectively, with CFD calculated streamlines (top half) and of computed shock locations (dashed lines in bottom half). The sting of the model is removed from the image and a rendering of the model is superimposed for illustrative purposes. Shock comparisons are made for $C_{T}$ of $0.5,1.0,2.0$, and 2.5, and the freestream flow is from left to right in these images. The CFD calculations begin in the PD jet plenum and calculate the flow through the jet nozzle. The CFD shock contour corresponds to the location where density begins to increase

Table 3 Comparison of sonic and supersonic shock standoff distance and jet penetration normalized by model diameter

\begin{tabular}{ccccc}
\hline \hline & \multicolumn{2}{c}{$\mathrm{SSD} / D_{\text {model }}$} & \multicolumn{2}{c}{ Jet Penetration $/ D_{\text {model }}$} \\
\cline { 2 - 5 }$C_{T}$ & Sonic & Supersonic & Sonic & Supersonic \\
\hline 0.5 & $0.14 \pm 21 \%$ & $0.14 \pm 21 \%$ & $0.19 \pm 16 \%$ & $0.28 \pm 11 \%$ \\
1.0 & $0.23 \pm 13 \%$ & $0.23 \pm 13 \%$ & $0.34 \pm 8.8 \%$ & $0.48 \pm 6.3 \%$ \\
1.5 & $0.32 \pm 9.4 \%$ & $0.32 \pm 9.4 \%$ & $0.43 \pm 7.0 \%$ & $0.64 \pm 4.7 \%$ \\
2.0 & $0.38 \pm 7.9 \%$ & $0.40 \pm 7.5 \%$ & $0.47 \pm 6.4 \%$ & $0.80 \pm 3.8 \%$ \\
2.5 & $0.45 \pm 6.7 \%$ & $0.49 \pm 6.1 \%$ & $0.53 \pm 5.7 \%$ & $0.86 \pm 3.5 \%$ \\
3.0 & $0.50 \pm 6.0 \%$ & $0.59 \pm 5.1 \%$ & $0.60 \pm 5.0 \%$ & $0.90 \pm 3.3 \%$ \\
\hline \hline
\end{tabular}



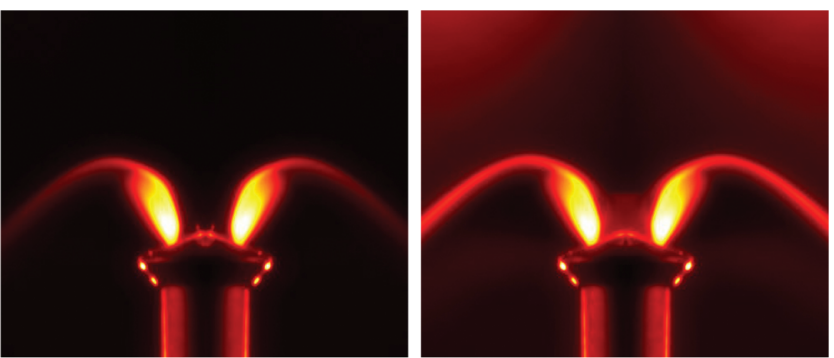

a) $\mathrm{C}_{\mathrm{T}}=1.5$, jet only seeded

b) $\mathrm{C}_{\mathrm{T}}=1.5$, full flow seeded

Fig. 6 Supersonic peripheral four-jet PD model, $M_{\text {jet }}=\mathbf{2 . 6 6}$, for two iodine seeding cases.

from freestream values along the aeroshell centerline. The bow shock profile can also be determined by the velocity streamline profile as the location where the slope of each streamline changes abruptly.

It is seen that, for the sonic case in Fig. 8, the PD jet freely expands from the nozzle exit until its bow shock, at which point the flow is swept out away from the model and downstream. Between the PD jets, the freestream flow compresses in a shock and the streamlines continue down to the model surface, at which point they reverse direction and follow the PD jet flow out from the model and downstream. Overall, there is good qualitative agreement between the experimental PLIIF images and the velocity streamlines and density contours calculated by LeMANS, especially between the PD jets upstream of the aeroshell.

The supersonic comparison shown in Fig. $\underline{9}$ also has good agreement between LeMANS calculations and the experimental measurement. Unlike the sonic case, CFD predicts a recirculation region near the PD jet and bow shock above the aeroshell centerline. There is good agreement between the CFD calculations and the experimental visualizations for $C_{T}$ of 0.5 and 1.0. However, for $C_{T}$ of
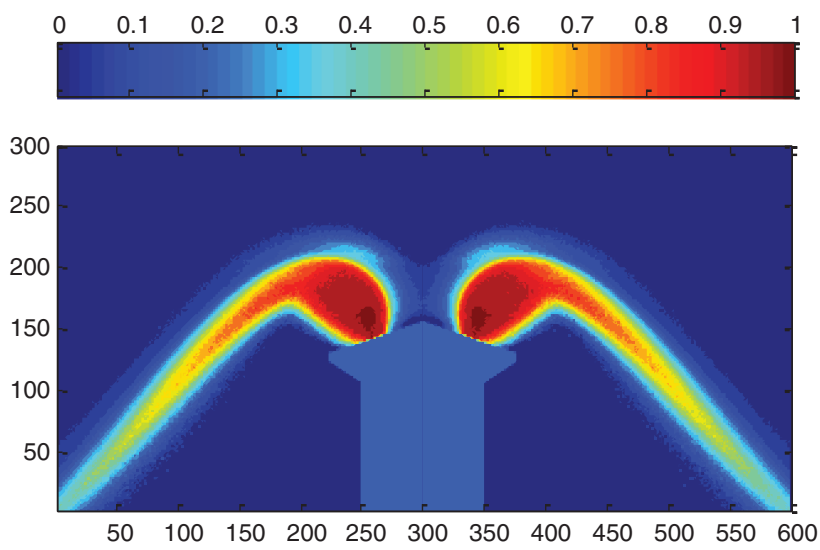

a) Sonic peripheral 4-jet PD model, $M_{\text {jet }}=1.0$
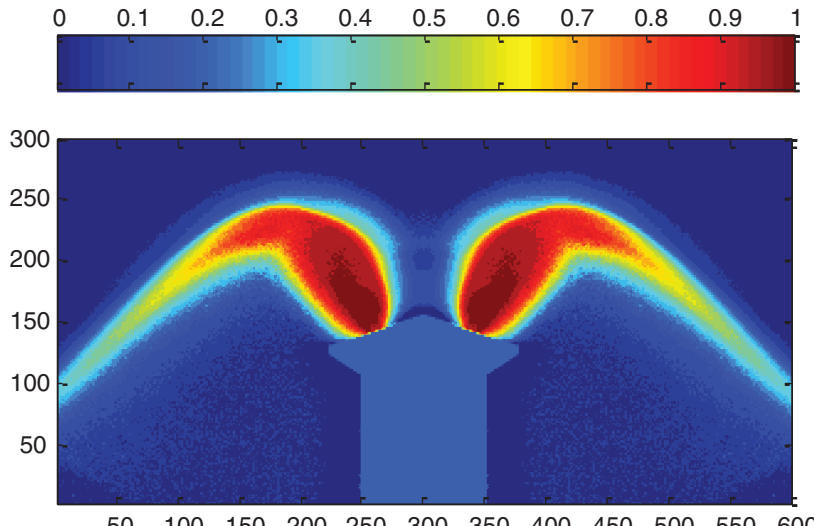

$\begin{array}{llllllllllll}50 & 100 & 150 & 200 & 250 & 300 & 350 & 400 & 450 & 500 & 550 & 600\end{array}$

b) Supersonic peripheral 4-jet PD model, $M_{\text {jet }}=2.66$

Fig. 7 Experimental four-jet PD mole fraction images, $C_{T}=1.5$.
2.0 and 2.5 , it is seen that the augmented shock location is farther downstream than in the measurement. One possible explanation is that, in the experiment, there may be a strong interaction of the aeroshell bow shock and the shock structure of the freestream freejet expansion. This interaction is not computed because the freestream flowfield is modeled by the Ashkenas and Sherman relationships [10], which give the Mach number as a function of axial distance and off-axis angle. As discussed earlier, the supersonic PD jets penetrate farther upstream than the sonic case, which may cause greater interaction than in the sonic case.

The shock standoff distance upstream of the aeroshell centerline versus $C_{T}$ for CFD calculations and the experimental results is compared in Fig. 10 for the sonic (a) and supersonic (b) PD models. Once again the shock standoff distance is normalized by the model diameter. For the CFD results, the shock location corresponds to the location where the density begins to increase above freestream values. The experimental shock location is calculated as described earlier by taking the point where fluorescence has increased $10 \%$ above the freestream fluorescence values. The error bars correspond to the uncertainty discussed earlier. Agreement between the numerical and experimental results increases from $12 \%$ and $17 \%$ for the sonic and supersonic cases, respectively, to within experimental uncertainty at $C_{T}=2.5$. The close agreement suggests that differences in the bow shock away from the aeroshell centerline
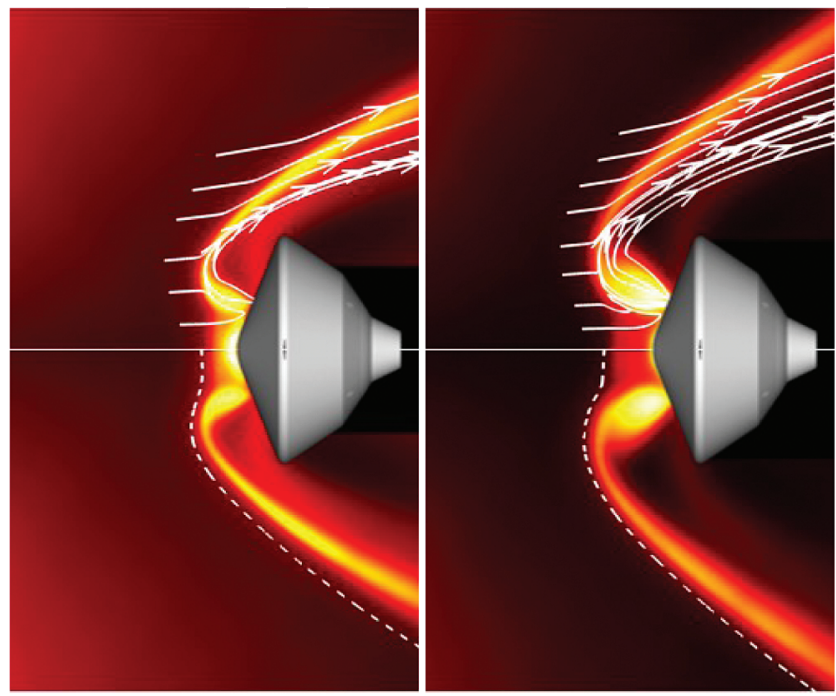

a) $\mathrm{C}_{\mathrm{T}}=0.5$

b) $\mathrm{C}_{\mathrm{T}}=\mathbf{1 . 0}$
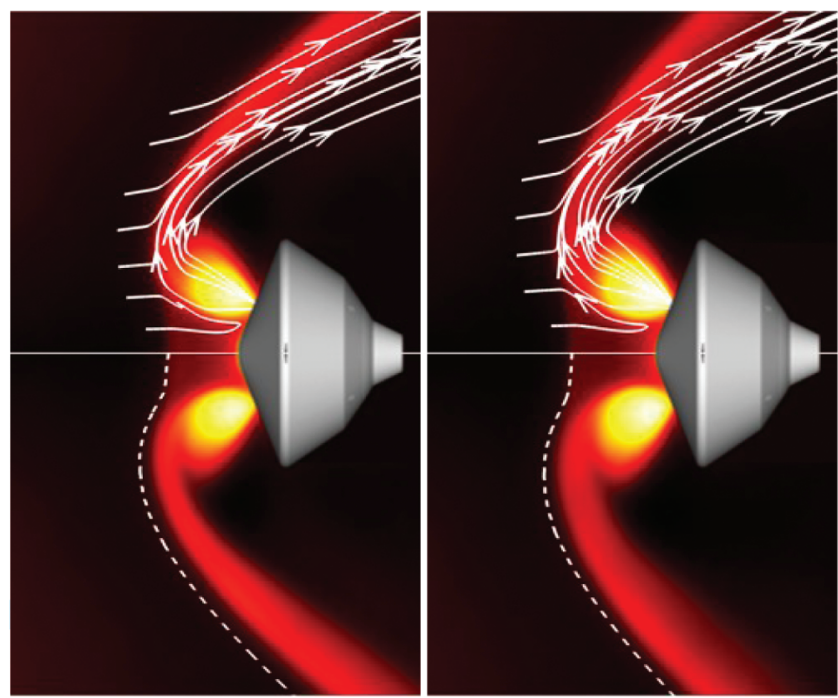

c) $\mathrm{C}_{\mathrm{T}}=\mathbf{2 . 0}$

d) $\mathrm{C}_{\mathrm{T}}=\mathbf{2 . 5}$

Fig. 8 Numerical calculation of streamlines overlaid on experimental visualizations $M_{\text {jet }}=1.0$. 

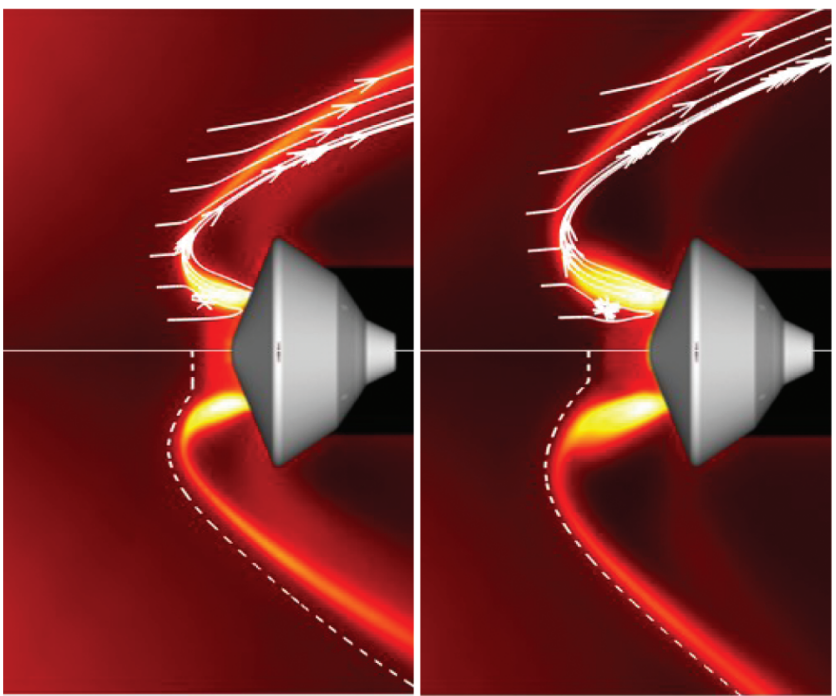

a) $\mathrm{C}_{\mathrm{T}}=0.5$

b) $\mathbf{C}_{\mathrm{T}}=\mathbf{1 . 0}$
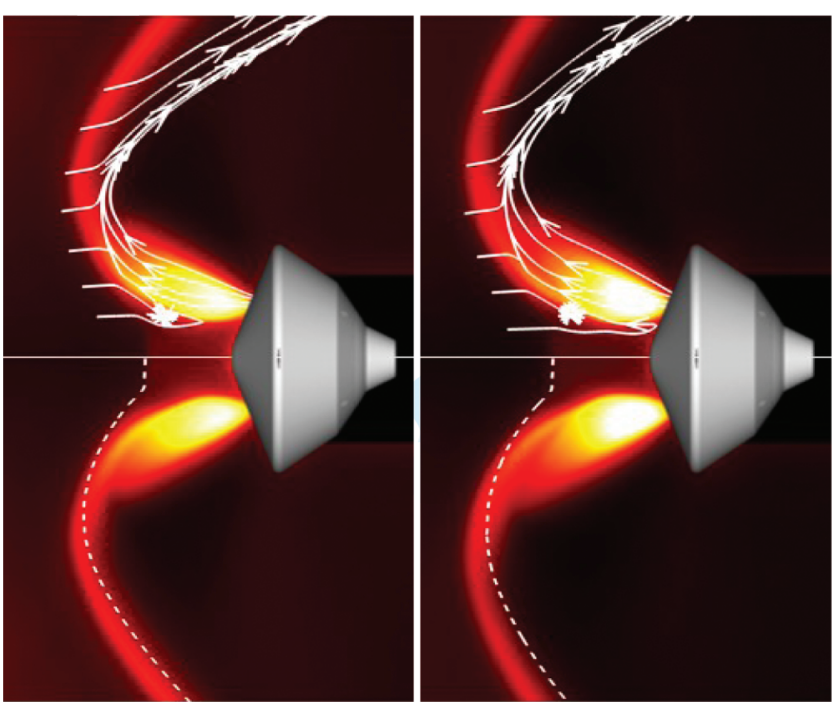

c) $\mathrm{C}_{\mathrm{T}}=\mathbf{2 . 0}$

d) $\mathrm{C}_{\mathrm{T}}=\mathbf{2 . 5}$

Fig. 9 Numerical calculation of streamlines overlaid on experimental measurement $M_{\text {jet }}=2.66$.

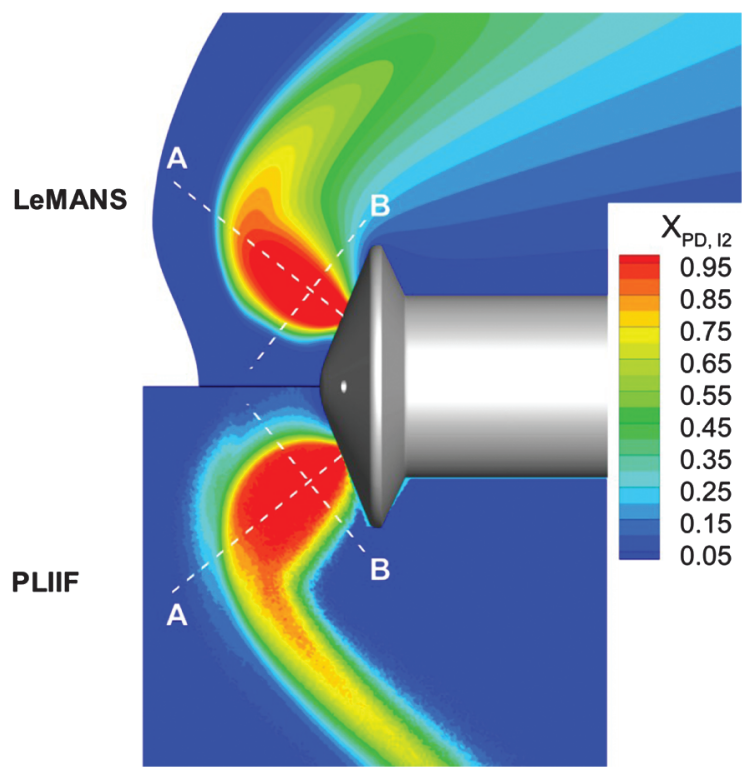

Fig. 11 Experimental PD jet mole fraction compared to CFD, $M_{\text {jet }}=1.0, C_{T}=1.5$.

observed in Fig. 9 may not have a significant effect on the flowfield properties near the aeroshell nose.

Quantitative comparisons between CFD and experimental PD jet mole fraction for the sonic model are shown in Fig. 11 for a $C_{T}$ of 1.5. Overall, there is good agreement between the CFD calculations and the experimental results. Discrepancies arise after the shoulder of the model where the CFD shows greater turning of the jet fluid around the model shoulder. These differences could be attributed to multiple factors, the first of which is continuum breakdown, which can occur for global Knudsen numbers greater than 0.05. As shown in [16], regions on the aftbody of the capsule approach a global Knudsen number of 1 . Another possible cause is due to the much heavier iodine molecule, which may not track the nitrogen molecules as the flow turns the aeroshell corner due to low bimolecular collision rate in the more rarefied region aft of the forebody. However, because the pressure is very low on the vehicle aftbody, this discrepancy will not have a significant impact on the calculation of the vehicle drag coefficient.

Figure 12 is a plot of the PD jet mole fraction versus the distance along lines A and B in Fig. 11 for the CFD calculations and PLIIF. Line A originates at the jet exit and follows along the jet centerline. The sharp drop in jet mole fraction across the jet boundary and shock

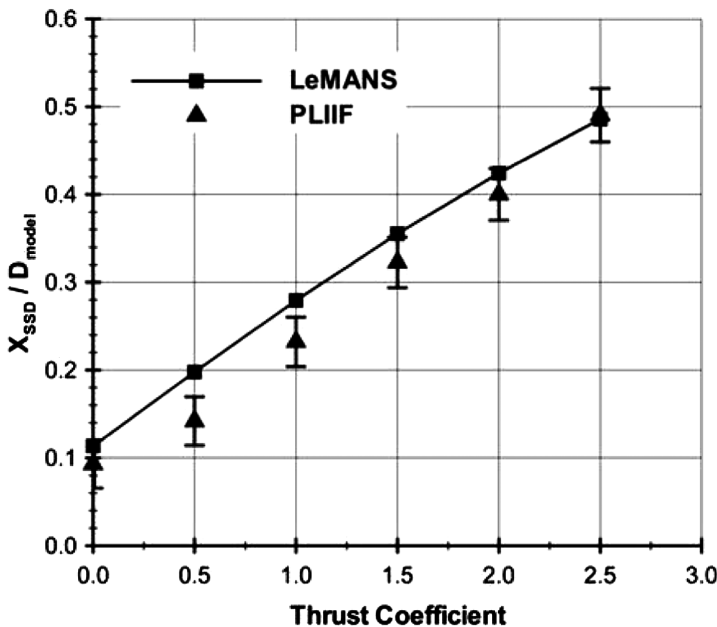

b) Supersonic model

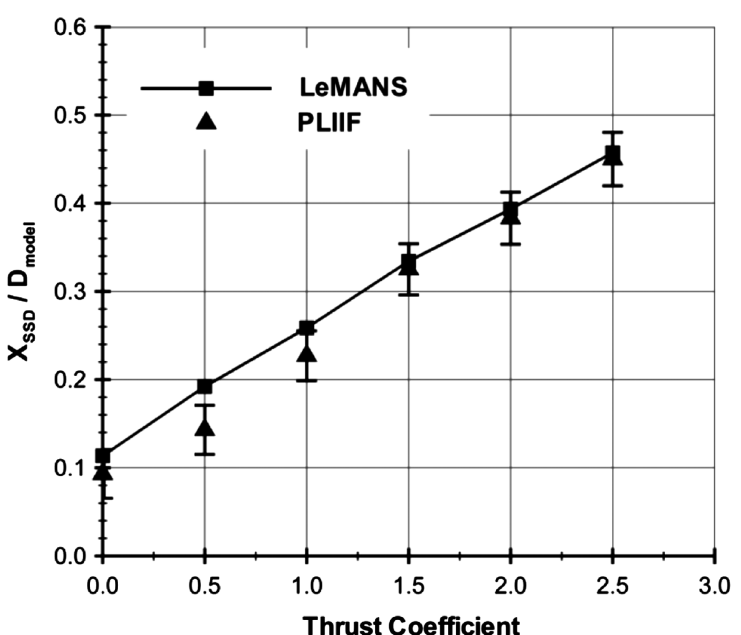

a) Sonic model

Fig. 10 Numerical calculation of $X_{\mathrm{SSD}} / D_{\text {Model }}$ combined with experimental visualization measurement. 


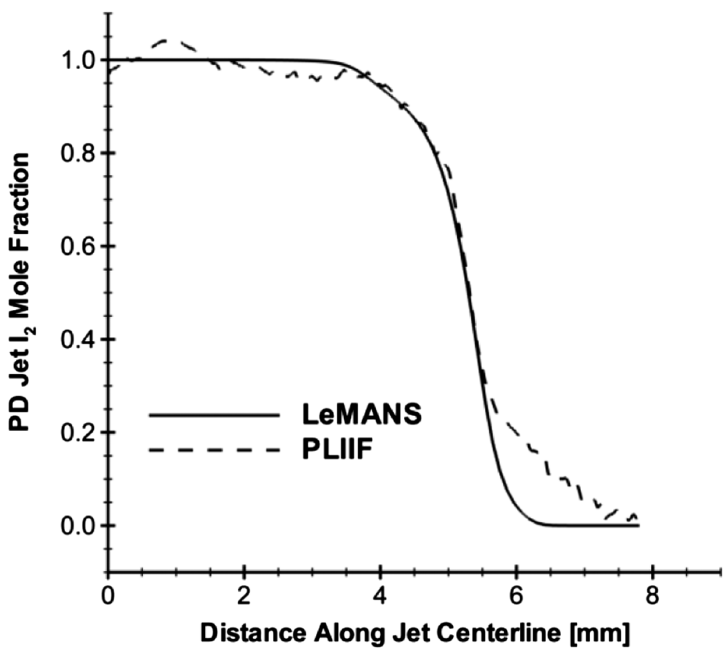

a) Line $\mathrm{A}$

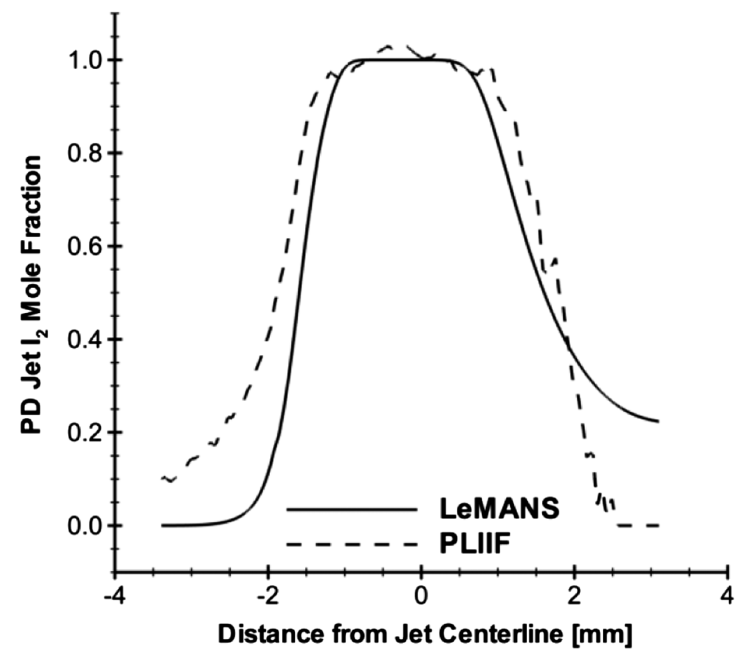

b) Line B

Fig. 12 Experimental jet mole fraction and CFD comparison.

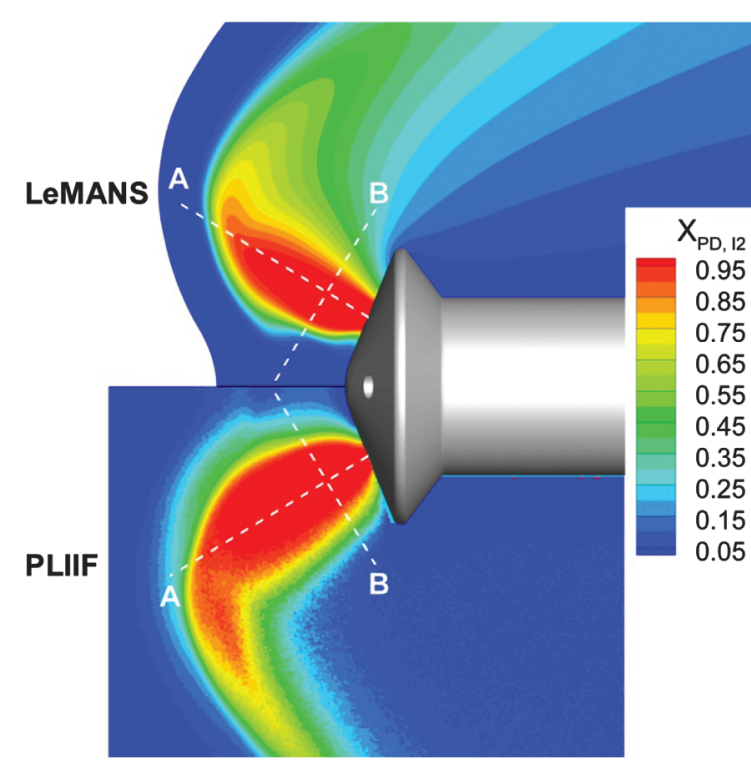

Fig. 13 Experimental PD jet mole fraction compared to CFD, $M_{\text {jet }}=2.66, C_{T}=1.5$.

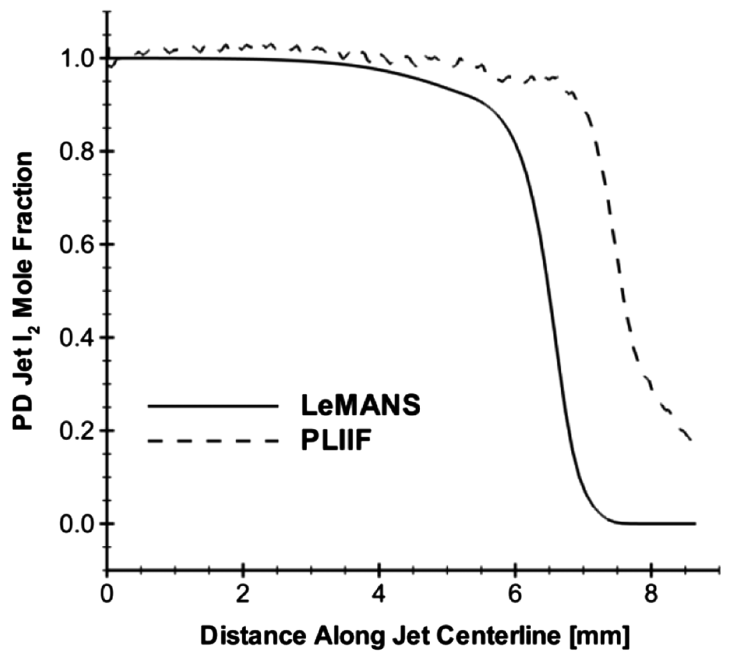

a) Line $A$ is clearly visible along line A. Line B is normal to line A and is located three nozzle exit diameters downstream of the exit. Increasing distance along line B indicates moving toward the shoulder of the model, as shown in Fig. 11. Once again, the jet mole fraction drops off sharply across the jet boundaries. Greater penetration into the freestream for the experimental case is seen for distances greater than $5.5 \mathrm{~mm}$ along line $\mathrm{A}$, and distances less than $2 \mathrm{~mm}$ along line $\mathrm{B}$. The increased fluid turning calculated around the model shoulder is seen in profile B for distances greater than $2 \mathrm{~mm}$. Overall, very good agreement is seen between the quantitative PLIIF mole fraction profiles and the CFD predictions.

Figure 13 is a quantitative comparison between CFD calculations and experimental results for the supersonic PD jet mole fraction at $C_{T}=1.5$. The experimental results have a broader PD jet, as shown by the PD jet mole fraction being close to unity for a greater portion of the flow about the jet centerline. Furthermore, like the sonic case, the LeMANS computations reflect a greater PD jet turning angle than the experimental results. The PD jet mole fraction versus distance for lines A and B are shown in Fig. 14. Line A, along the jet centerline, shows good agreement; however, greater jet penetration is seen for the experiment. Line B also shows good agreement between calculations and experiment (located two jet-exit diameters from the jet exit); however, the broader PD jet profile is seen in the experimental results as well as the increased jet fluid around the aeroshell shoulder in the CFD result.

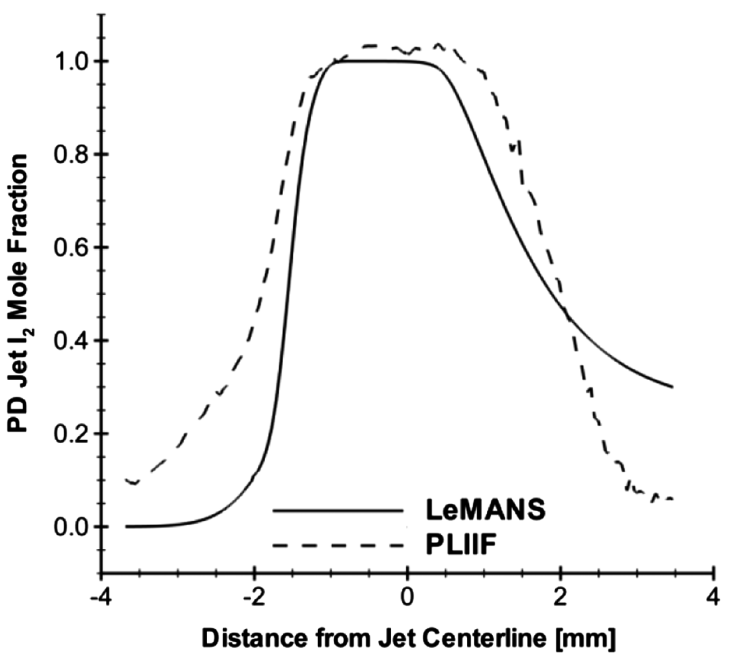

b) Line B

Fig. 14 Experiment jet mole fraction and CFD comparison. 


\section{Conclusions}

Experimental qualitative planar laser-induced iodine fluorescence flow visualizations and quantitative mole fraction measurements have been presented and discussed for multiple sonic and supersonic peripheral jets on a Mars Science Laboratory frontal aeroshell at Mach 12. Experimental results for the range of thrust coefficients from 0.5 to 3.0 , in increments of 0.5 , have demonstrated that a bow shock between the jets is preserved. As thrust coefficient increases, the shock standoff distance increases for both sonic and supersonic models and is roughly equivalent for thrust coefficient less than 2.0. However, for thrust coefficient greater than 2.0, the shock standoff for the supersonic model is greater than the sonic, being $18 \%$ greater for thrust coefficient of 3.0. Jet penetration also increases for all thrust coefficients and was greater for the supersonic case than the sonic, resulting in approximately $50 \%$ greater jet penetration for the supersonic case at a thrust coefficient of 3.0. Further differences that are observed include a greater propulsive decelerator jet turning angle and broader jet boundary in the sonic case.

Computational fluid dynamic calculations appear to capture the major flow characteristics seen in the experimental results. Overall, there is good qualitative agreement between shock structure and calculated streamlines and shock location for the sonic propulsive decelerator model for thrust coefficient ranging from 0.5 to 2.5. Experimental results and calculations also have good agreement in the shock location directly above the nose of the aeroshell for the supersonic test cases; however, discrepancies were observed in shock location away from the aeroshell, as well as the greater interation of the bow shock with freestream barrel shock at higher thrust coefficients in the supersonic test cases. Quantitative mole fraction calculations are shown to be in good agreement with the experimental results through the propulsive decelerator jet core and on a crosssectional cut through the jet core for both sonic and supersonic test cases, although there is some difference around the model shoulder, where calculations predict greater fluid turning in both cases, and the supersonic jet penetrates farther into the freestream experimentally.

\section{Acknowledgments}

This work is supported by NASA Hypersonics Project NRA Grant NNX08AH37A, with Bryan Palaszewski from the NASA John H. Glenn Research Center at Lewis Field as the technical monitor, a NASA Graduate Student Researchers Program Fellowship (Codoni), and a National Science Foundation Fellowship (Reed). The University of Michigan would like to acknowledge the use of supercomputers through the NASA Advanced Supercomputing Division.

\section{References}

[1] Braun, R. D., and Manning, R. M., "Mars Exploration Entry, Descent, and Landing Challenges," Journal of Spacecraft and Rockets, Vol. 44, No. 2, 2007, pp. 310-323. doi: $10.2514 / 1.25116$

[2] Dwyer-Cianciolo, A. M., Davis, J. L., Komar, D. R., Munk, M. M., Samareh, J. A., Powell, R. W., Shidner, J. D., Stanley, D. O., Wilhite, A. W., Kinney, D. J., McGuire, M. K., Arnold, J. O., Howard, A. R., Sostaric, R. R., Studak, J. W., Zumwalt, C. H., Llama, E. G., Casoliva, J., Ivanov, M. C., Clark, I., and Sengupta, A., "Entry, Descent and Landing Systems Analysis Study: Phase 1 Report," NASA TM-20100000002009, July 2010.
[3] Korzun, A. M., Braun, R. D., and Cruz, J. R., "Suvery of Supersonic Retropropulsion Technology for Mars Entry, Descent, and Landing," Journal of Spacecraft and Rockets, Vol. 46, No. 5, 2009, pp. 929-937. doi: 10.2514/1.41161

[4] Berry, S. A., Rhode, M. N., Edquist, K. T., and Player, C. J., "Supersonic Retropropulsion Experimental Results from the NASA Langley Unitary Plan Wind Tunnel," AIAA Paper 2011-3489, June 2011.

[5] Jarvinen, P. O., and Adams, R. H., "The Aerodynamic Characteristics of Large Angled Cones with Retrorockets," NASA, Contract No. NAS-7576, Feb. 1970.

[6] Jarvinen, P. O., and Adams, R. H., "The Effects of Retrorockets on the Aerodynamic Characteristics of Conical Aeroshell Planetary Entry Vehicles," AIAA Paper 1970-219, Jan. 1970.

[7] Keyes, J. W., and Hefner, J. N., "Effect of Forward-Facing Jets on Aerodynamic Characteristics of Blunt Configurations at Mach 6," Journal of Spacecraft and Rockets, Vol. 4, No. 4, 1967, pp. 533-534.

[8] Korzun, A. M., and Braun, R. D., "Performance Characterization of Supersonic Retropropulsion for High-Mass Mars Entry Systems," Journal of Spacecraft and Rockets, Vol. 47, No. 5, 2010, pp. 836-848. doi: $10.2514 / 1.49803$

[9] Staack, D., McDaniel, J. C., Glass, C. E., and Miller, C., "Experimental Study of Interacting Rarefied and Continuum Flows," AIAA Paper 2001-2762, June 2001.

[10] Ashkenas, H., and Sherman, F. S., "The Structure and Utilization of Supersonic Free Jets in Low Density Wind Tunnels," edited by de Leeuw, J. H., Rarefied Gas Dynamics, Vol. 2, Academic Press, New York, 1966, pp. 84-105.

[11] Cecil, D. E., "Planar Laser-Induced Iodine Fluorescence Measurements in Rarefied Hypersonic Flow Over a Reaction Control Jet Model in a Free Jet Wind Tunnel," M.S. Thesis, Univ. of Virginia, Charlottesville, VA, Jan. 2004

[12] Cecil, D. E., and McDaniel, J. C., "Planar Laser-Induced Iodine Fluorescence Measurements in Rarefied Hypersonic Flow," edited by Capitelli, M., Rarefied Gas Dynamics: 24th International Symposium, American Institute of Physics, New York, 2005, pp. 1325-1330.

[13] Hartfield, R. J., Hollo, S. D., and McDaniel, J. C., "Planar Temperature Measurement in Compressible Flows Using Laser-Induced Iodine Fluorescence," Optics Letters, Vol. 16, No. 2, 1991, pp. 106-108. doi:10.1364/OL.16.000106

[14] Reed, E., Codoni, J., McDaniel, J. C., Alkandry, H., and Boyd, I. D., "Investigation of the Interactions of Reaction Control Systems with Mars Science Laboratory Aeroshell," AIAA Paper 2010-1558, Jan. 2010.

[15] Hiller, B., "Combined Planar Measurments of Velocity and Pressure Fields in Compressible Gas Flows Using Laser-Induced Iodine Fluorescence," Ph.D. Dissertation, Stanford Univ., Stanford, CA, Nov. 1986.

[16] Alkandry, H., Boyd, I. D., Codoni, J. R., Reed, E. M., and McDaniel, J. C., "Numerical Investigation of Multi-Nozzle Propulsive Deceleration Jets for Mars Entry Aeroshells," Journal of Spacecraft and Rockets, Vol. 50, No. 6, 2013, pp. 1196-1206. doi:10.2514/1.A32455

[17] McGhee, R. J., "Effects of a Retronozzle Located at the Apex of a $140^{\circ}$ Blunt Cone at Mach Numbers of 3.00, 4.50, and 6.00," NASA TN-D6002, Jan. 1971.

[18] McDaniel, J. C., Codoni, J. R., Reed, E. M., Alkandry, H., and Boyd, I. D., "Propulsion Deceleration Studies Using Planar Laser-Induced Iodine Fluorescence and Computational Fluid Dynamics," Journal of Spacecraft and Rockets, Vol. 50, No. 4, 2013, pp. 771-780. doi:10.2514/1.A32328

M. MacLean Associate Editor 